\title{
Gray-scale graphics using dot matrix printers
}

\author{
CHARLES W. WHITE, EDWARD M. BRUSSELL, \\ TERRENCE T. WILLIAMS, and STANISLAW J. RÖG \\ Concordia University, Montréal, Québec, Canada
}

\begin{abstract}
Gray-scale graphics can be printed on conventional dot matrix printers by varying the dot density, rather than the dot intensity. The technique described here takes advantage of the high-contrast print possible with these printers and the increased resolution available using compressed character sets. The reflectance of individual characters and symbols was measured with a photometer, and a character set was selected that increased from dark to light in equal logarithmic steps of reflectance. Various criteria for choosing dot patterns are discussed, and the technique is applied to two-dimensional visual field maps.
\end{abstract}

In the behavioral sciences, there are many applications for two-dimensional data displays. In vision research, for example, psychophysical measurements are often made at various points in the visual field. Other examples include locating labeled regions in brain tissue by tomographic techniques (Arai, Kobayashi, Ikeda, Nagao, Ogihara, \& Kosaka, 1983) and directional sensitivity of moths to ultrasound (Roeder, 1965), as well as standard research tools such as thermograms (Nagayama, Ohnuki, \& Kanosue, 1981). Although it is possible to display such data as combinations of one-dimensional graphs, many important features of the results are averaged out in one dimension. The interesting details may be visible only in two-dimensional displays. The purpose of this article is to consider some of the criteria that enter into deciding how to display two-dimensional data and to suggest a general method for solving some of the practical problems involved in creating such displays.

The application that prompted our current interest arose from a research project on visual sensitivity in multiple sclerosis (Brussell, White, Bross, Mustillo, \& Borenstein, 1981/82; White, Brussell, Overbury, \& Mustillo, 1983). One of the characteristics of multiple sclerosis (MS) is that the temporal sensitivity is selectively impaired in different areas of the visual field. In order to find the affected areas in MS patients, psychophysical measurements were made of temporal resolution at various points in the visual field. A computer mapping program (Dougenik \& Sheehan, 1975) converted the data points into a two-dimensional map. This computer mapping package, called SYMAP, is available on many academic and research computing systems. It is a very flexible package, and it allows selection among many differ-

The research described here was supported by Grant A7482 from the Natural Sciences and Engineering Research Council of Canada and Grant EQ-1135 from les Fonds F.C.A.C. pour l'aide et le soutien à la recherche du Québec. Reprint requests should be addressed to the first author at the Department of Psychology, Concordia University, 7141 Sherbrooke Street, Montréal, Québec, Canada H4B 1R6. ent plotting options, but it has one drawback for our purposes-it is designed for line printer output.

There are many reasons why line printers are not suitable for gray-scale graphics: (1) There is little standardization of line printers at different computer installations. Since character sets differ from one manufacturer to another and from one line printer model to another, the density or reflectance values for the characters used to print the map may not be those expected by the program. (2) In remote time-sharing applications, it is important to be able to view the results of the mapping program at the terminal, without having to wait for the line printer output from the central computer. It is difficult to obtain accurate density plots using a remote terminal, because different remote terminals use different sets of printing characters. (3) In order to increase the range of reflectances available from line printers, the standard character set used by SYMAP includes overprinted characters, but overprinting is impossible on some terminals or printers and either difficult to implement or unbearably slow in execution on others. (4) Finally, for many purposes, the standard printing densities on most line printers (10 characters/in. and 6 or 8 lines/in.) are too widely spaced for depicting uniform gray levels.

\section{METHOD}

The basic method for determining character set assignments for density maps is straightforward, but tedious. First, print some samples of blocks of repeated characters. Next, measure the reflectance of the samples and then select an appropriate subset of the characters to be used as printing symbols.

The details of our application of this approach are as follows: First, we wrote a program to print successive blocks of all the printable characters on the printing terminal (Digital Equipment Corporation, DECwriter Model LA-120). A simple program to print the character blocks appears in Table 1. (The program is written in Applesoft BASIC for the Apple II series of microcomputers, but the translation to other microcomputer systems should be straightforward.)

Each character block was printed at 16.5 characters/in. and 12 rows/in. with a new black ribbon. The characters were spaced as closely as possible in order to enhance the maximum 
Table 1

Applesoft Program to Print Blocks of Characters

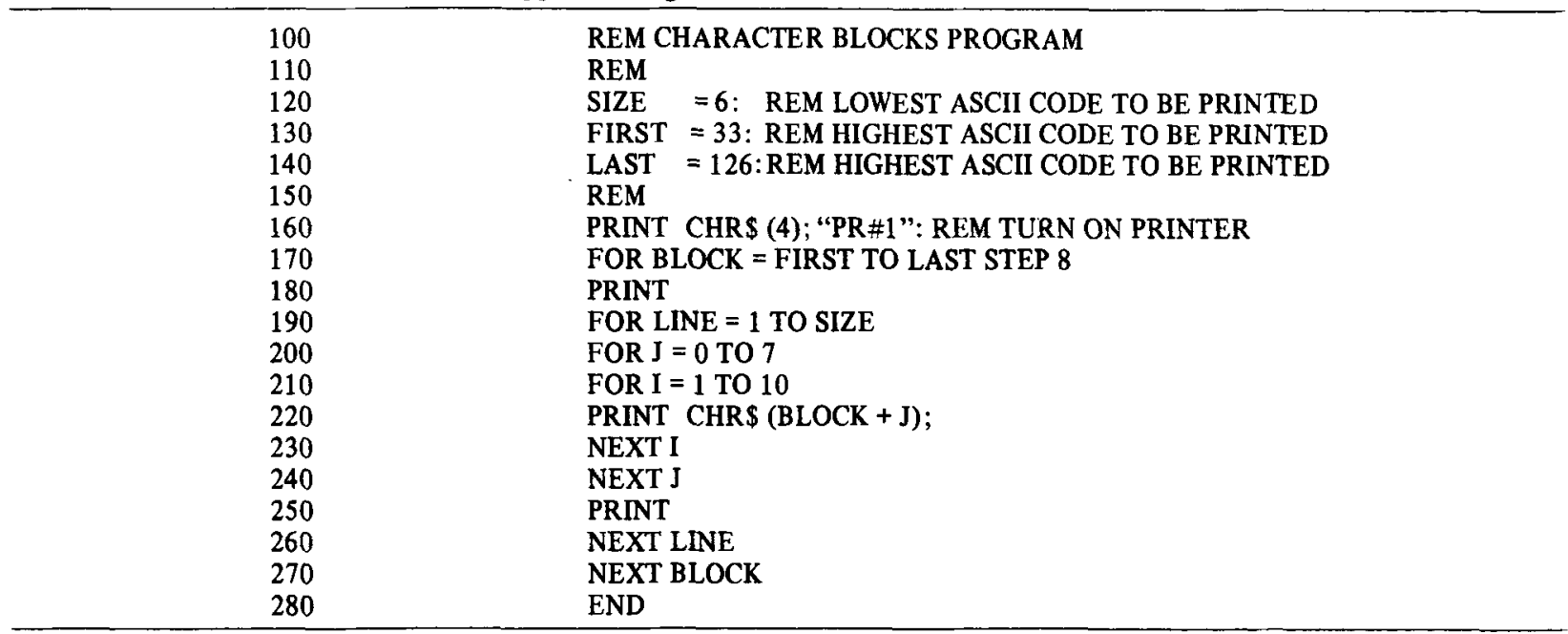

Table 2

A Set of Printing Symbols for a Seven-Level Gray Scale

\begin{tabular}{cclcc}
\hline & $\begin{array}{c}\text { Printing } \\
\text { Level } \\
\text { Symbol }\end{array}$ & Description & \multicolumn{1}{c}{$\begin{array}{c}\text { Log } \\
\text { Reflectance Reflectance }\end{array}$} \\
\hline 7 & 0 & "at" & .42 & -.381 \\
6 & $\%$ & per cent & .48 & -.318 \\
5 & J & capital J & .56 & -.251 \\
4 & $>$ & greater than & .62 & -.211 \\
3 & $"$ & quotation mark & .70 & -.152 \\
2 &, & apostrophe & .79 & -.101 \\
1 & & space & .89 & -.051 \\
\hline
\end{tabular}

contrast range. The next step was to measure the reflectance of each sample block of characters, using a calibrated reflex-viewing photometer (Spectra spotmeter, Model UBD). The reflectance values were then tabulated in order of increasing reflectance.

The choice of the subset of plotting symbols was constrained by several considerations. For example, the numerals were excluded because they were to be used for plotting numerical values on the map. Also, since most alphabetic characters would have interfered with labels on the map, punctuation and nonalphabetic symbols were selected whenever possible. For the present application, the intention was to scale the reflectances of the symbols such that equal scale steps appeared as equal steps of lightness or darkness. Lightness scaling is a notoriously difficuit task in visual psychophysics because the perception of lightness and darkness is affected by many variables, such as texture, contour, and luminance gradients. Even when such extraneous factors are ignored, however, the formulas for translating photometric reflectance values into perceptual lightness scales are complicated. For example, the CIE Colorimetry Committee proposed the following cube-root formula (Wyszecki, 1974): $L^{*}=\left[25(100 Y)^{1 / 3} / Y_{0}\right]-16$, where the lightness value, $L^{*}$, is a function of the stimulus luminance factor, $Y$, and the luminance factor of a perfect reflecting diffuser, $Y_{0}$.

In order to be very precise about constructing equal lightness steps, one could use such specialized formulas. However, in the application described here, we simply selected symbols that had approximately equally spaced reflectances when plotted on a logarithmic scale. The symbols and their reflectance values appear in Table 2.

\section{RESULTS}

In order to illustrate the method, a seven-level gray scale was printed (shown in Figure 1). The lightness differences between adjacent levels should appear approximately the same across the scale. The inset in Figure 1 is a magnified portion of the character block printed with the percent symbol $(\%)$, which illustrates the overlapping of the printed rows of characters.

Another example of a seven-level gray-scale map is shown in Figure 2. It is a visual field map of temporal resolution thresholds for a patient with multiple sclerosis.

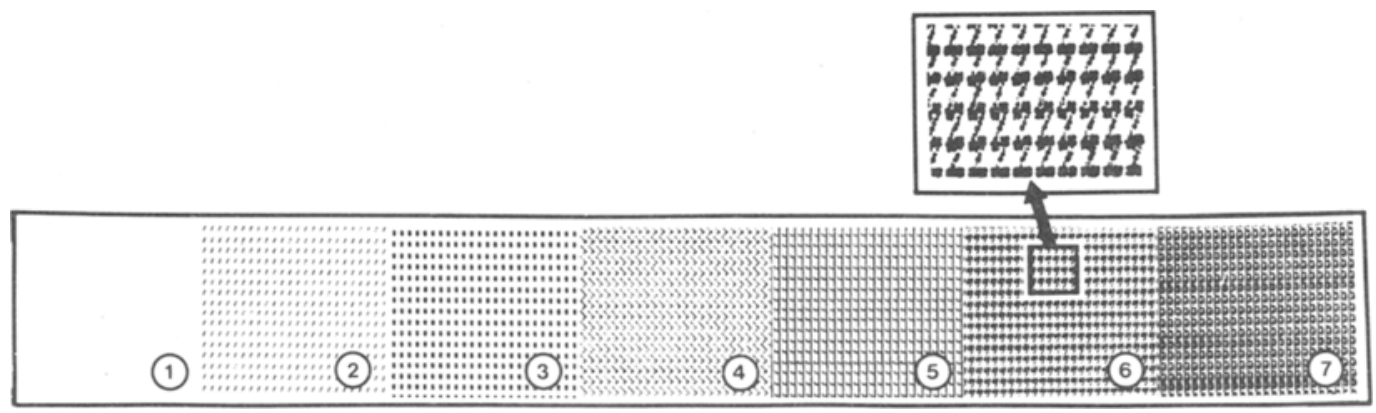

Figure 1. Seven-level gray scale printed on a dot matrix printer. The inset illustrates a magnified view of one of the printing symbols. 


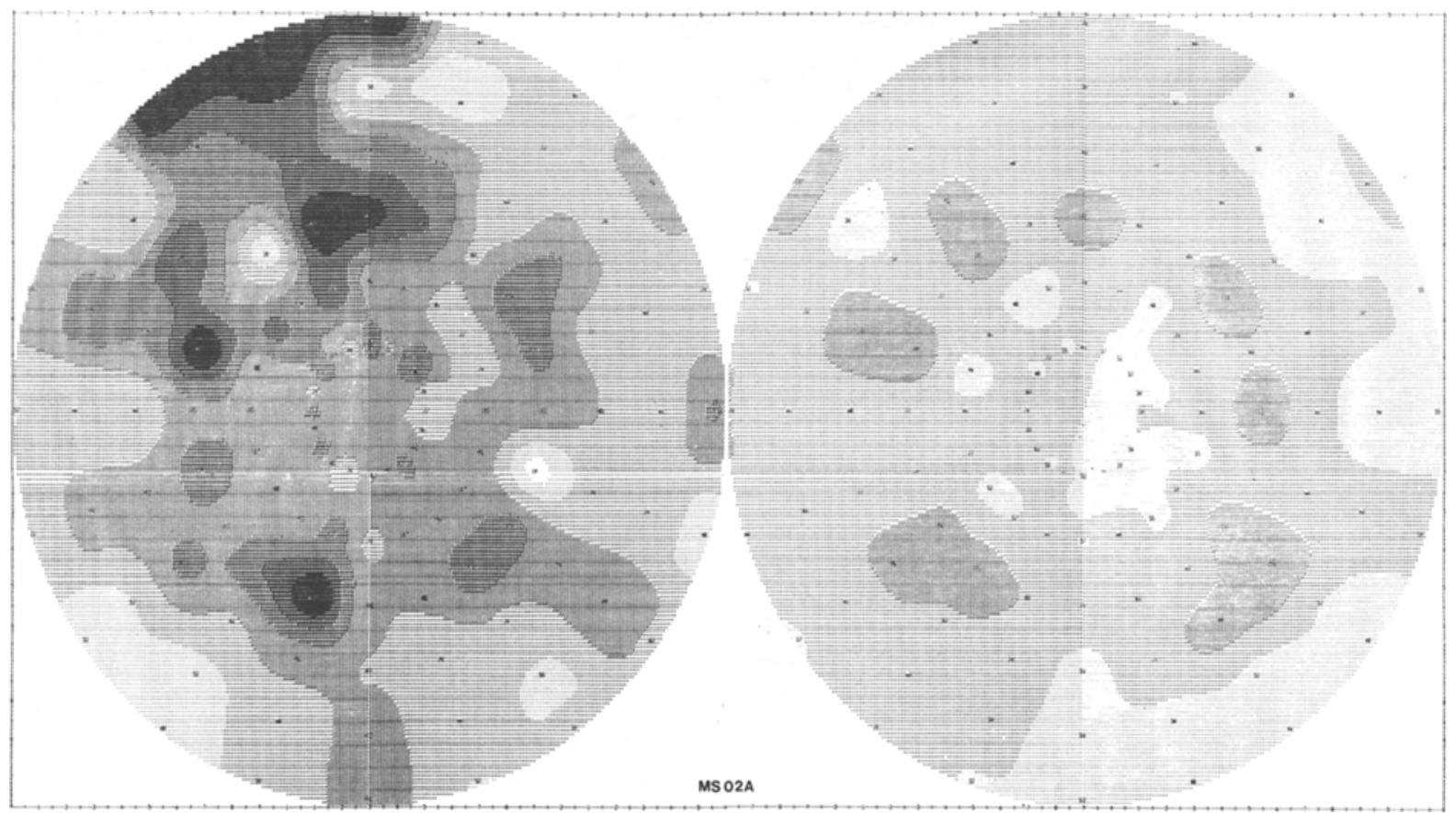

Figure 2. Visual field map of temporal resolution for a patient with multiple sclerosis. The darker the map, the worse is the temporal resolution in the corresponding region of the patient's visual field. The circular field for each eye subtends a visual angle of $\mathbf{4 0} \mathrm{deg}$, and the central region of each eye's field has been expanded. See text for details.

The data from the observer's left eye are shown on the left, and the data from the right eye are on the right. The coordinate system is in visual field, rather than retinal, coordinates. The circular field for each eye subtends a visual angle of $40 \mathrm{deg}$, and the central area of each field has been expanded by logarithmic scaling of the radial distance in order to emphasize the functional significance of the foveal region and to increase the spatial resolution of the foveal mapping. Additional details are explained in Brussell et al. (1981/82) and White et al. (1983).

\section{DISCUSSION}

In the application described here, the gray-scale technique was found to be an effective and sensitive method for illustrating subtle spatial variations in the visual field data. This was accomplished while holding constant the contrast of individual dots and manipulating the density of the printed dots per unit of area. One advantage to this technique, in addition to the speed with which data can be displayed, is that it facilitates reproduction by techniques that ordinarily distort gray levels, such as photocopying.

One of the difficulties encountered in applying this method is the variety of dot matrices used by different printers to generate characters and symbols. Obviously. reflectance values calculated for one printer's character set differ from those for another set of dot patterns. A possible solution could be to use the user-definable character sets that are available on some of the newer dot matrix printers. If a calibrated dot matrix pattern could be defined and installed, then it would be possible to print the graphics on various printers without distorting the gray scale.

Although this method of producing gray-scale graphics has been applied to dot matrix printers, a similar approach should be possible with computer graphic displays. Provided that the gray scale is produced by manipulating dot density rather than dot intensity, then high contrast reproduction of video or CRT graphics should be possible.

\section{REFERENCES}

Arai, H., Kobayashi, K., IKeda, Y., Nagao, Y., Ogihara, R., \& KoSAKA, K. (1983). A computed tomography study of Alzheimer's disease. Journal of Neurology, 229, 69-78.

Brusgell, E. M., White, C. W., Bross, M., Mustillo, P., \& Borenstein, M. (1981/82). Multi-flash campimetry in multiple sclerosis. Current Eye Research, 1, 671-677.

Douannik, J. A., \& Sheehan, D. E. (1975). SYMAP user's reference manual. Cambridge, MA: Harvard University Laboratory for Computer Graphics and Spatial Analysis.

Nagayama, T., Ohnuti, Y., \& Kanosue, K. (1981). Fall in skin temperature during exercise observed by thermography. Japanese Journal of Physiology, 31, 757-762. 
Roeder, K. (1965). Moths and ultrasound. Scientific American, 212(4), 94-102.

White, C. W., Brussell, E. M., Overbuty, O., \& Mustillo, P. (1983). Assessment of temporal resolution in multiple sclerosis by multi-flash campimetry. In O. M. Breinin \& I. M. Siegel (Eds.), Advances in diagnostic visual optics. Berlin: SpringerVerlag.
Wyszecki, G. (1974). CIE Colorimetry Committee-Working program on color differences. Journal of the Optical Society of America, 64, 896-897.

(Manuscript received November 28, 1983; revision accepted for publication January $23,1984$. ) 